SGM Special

\section{Lecture}

Fred Griffith Prize

Lecture 2007

Delivered at the 161st meeting

of the SGM, 4 September 2007

Correspondence

E. Richard Moxon

richard.moxon@

paediatrics.ox.ac.uk

\title{
Bacterial variation, virulence and vaccines
}

\section{E. Richard Moxon}

\begin{abstract}
Weatherall Institute of Molecular Medicine, John Radcliffe Hospital, Headington, Oxford OX3 9DS, UK
\end{abstract}

\begin{abstract}
Research into Haemophilus influenzae, a commensal and pathogen of humans, has resulted in major scientific contributions to biology. The first endonucleases (restriction enzymes), which paved the way for the new genetics, and the DNA used to obtain the first complete genome sequence of a free-living organism were obtained from $H$. influenzae. Prevention of invasive bacterial infections of infants, such as meningitis, has been achieved using a novel class of vaccines, of which the glycoconjugates of $H$. influenzae were the first to be licensed. Originally fallaciously proposed to be the aetiological agent of epidemic influenza, now known to be caused by a virus, $H$. influenzae is a pathogen of global public health importance. Research into the pathogenesis of the infections it causes (for example, meningitis, septicaemia, pneumonia and otitis media) are case studies in understanding the molecular basis of the variation in gene expression and gene sequences that are critical to its commensal and virulence behaviour and for the strategies that can be pursued to prevent $H$. influenzae diseases through vaccines.
\end{abstract}

\section{Introduction}

In the First Griffith Memorial Lecture, entitled 'Genetic Transformation: a Retrospective Appreciation', W. Hayes described Fred Griffith '....as a shy and reticent man, whose quiet kindly manner, and his devotion to his job, made him a lovable personality to those few who got to know him. Outside his work he found pleasure in ski-ing and in walking on the Sussex Downs where he had built a cottage. Like his elder brother Stanley, who died only a few days before him, he was a medical microbiologist whose primary and abiding interest, and his life's work, was the epidemiology of infectious disease. He believed that a proper understanding of epidemiological problems could come only from more detailed and discriminating knowledge of infectious bacterial species, and of the nature of bacterial virulence and variation' (Hayes, 1966).

Griffith's discovery of natural transformation in the pneumococccus inspired the discovery of the role of DNA, the transforming principle (McCarty, 1985), in heredity. It is a matter of profound importance and fascination that information specifying the variations in shape, size, colour, and other attributes of organisms are stored in and transmitted to progeny by sequences of nucleic acids. But the diversity of life forms is extraordinary and it is the heritability of differences in the nucleotide sequence of DNA or RNA that underlies the evolution of natural variation in all living organisms. In this lecture, I would like to return to the themes of virulence and variation that featured in William Hayes' inaugural lecture but in the context of Haemophilus influenzae, which has been my major research interest. Why so much time devoted to the biology of one bacterium? After all, one could argue that many of its individual properties will be special and not generally applicable to other organisms. However, I hope to persuade you that natural variation of $H$. influenzae is the key to understanding its success as a commensal and its potential as a pathogen, and that these insights may have broader implications for understanding the commensal and virulence behaviour of other bacterial species. Further, antigenic diversity is highly relevant to our efforts to prevent disease through vaccines.

First, some brief introductory comments about $H$. influenzae (Hi). Griffith's experiments on transformation secured a firm place in biomedical history for the pneumococcus. I suggest that $H i$ also deserves a place on the podium alongside its illustrious co-inhabitant of the human upper respiratory tract. For example, the endonucleases obtained from $\mathrm{Hi}$ catalysed the 'new genetics', and subsequently the genome sequence of $H i$ strain Rd revolutionized biology and ushered in the genomic era. Other facts worthy of mention are that $\mathrm{Hi}$ was only the second example of natural transformation, and coincidentally the first demonstration of conferring antibiotic resistance (to streptomycin) through DNA (Alexander \& Leidy, 1953). Further, one of the earliest documented examples of the use of a sulphonamide in clinical practice was the treatment of a severe case of $\mathrm{Hi}$ invasive disease (Carithers, 1974). Some important milestones in Hi research are summarized in Table 1.

\section{$H$. influenzae is a specialist commensal and pathogen of humans}

After the confusion concerning the role of $\mathrm{Hi}$ as a primary cause of influenza was settled (Smith et al., 1933), there emerged a clear perspective of its capacity to cause a variety of infections as a primary pathogen and also its role as a 
Table 1. Milestones in $H$. influenzae research

1889 Pfeiffer's bacillus: mistaken aetiology of influenza
1923 Officially named Haemophilus influenzae; a serious pathogen, especially of children
1933 Margaret Pittman describes the capsular serotypes of H. influenzae
1978 Nobel Prize awarded to Ham Smith, Dan Nathans and Werner Arber for the discovery and exploitation of restriction enzymes
(endonucleases) obtained from H. influenzae
1982 Licensure of the H. influenzae glycoconjugate vaccines for the prevention of invasive diseases caused by type b strains
$1995 \quad$ Completion of sequence of H. influenzae strain Rd, the first complete genome sequence of a free-living organism

secondary invader following a variety of virus infections. But what was not adequately appreciated until much later was that $\mathrm{Hi}$ is first and foremost an extremely common commensal of the human upper respiratory tract of normal, healthy individuals. Nonetheless, through contiguous spread from the nasopharynx, commensal $\mathrm{Hi}$ commonly become pathogenic and cause otitis media, sinusitis and pneumonia, especially in individuals where the clearance mechanisms of the respiratory tract are compromised. More rarely, Hi can invade the blood, resulting in bacteraemia, even septicaemia, and disseminate to distant sites such as the meninges or the synovia of joints. In general, these serious invasive (bacteraemic) infections are caused predominantly by encapsulated strains and, remarkably, these systemic infections are almost invariably caused by type b strains. For poorly understood reasons, the type b capsular polysaccharide, ribosyl-ribitol phosphate, confers heightened virulence properties upon $H i$, pathogenic properties that are denied to strains that lack this capsule or synthesize one of five other structurally defined capsular polysaccharides (Moxon \& Vaughn, 1981; Zwahlen et al., 1989). Despite the importance (from the public health perspective) of its pathogenic properties, survival of the species $H i$ is not dependent upon the ability to cause disease because the vast majority of bacteria that enter the blood or spread to the lung or middle ear come literally to a dead end. Rather, the most important evolutionary factors are those that determine its success as a commensal - its capability to reside in the nasopharynx and spread between humans. Disease is apparently incidental or accidental to the life history of $\mathrm{Hi}$.

\section{$H$. influenzae pathogenicity is a function of its fitness}

In considering the evolution of $\mathrm{Hi}$ virulence, it makes sense to consider that the biological basis of its pathogenic behaviour stems from the strategies adopted by $\mathrm{Hi}$ to maintain its basic reproductive rate through residence within and spread between its obligate hosts, humans. Although complex, the biology of $\mathrm{Hi}$, indeed of any organism, can be simplified: either you survive or you do not. Over time, any organism must face the challenges of its immediate environments (animate or inanimate) to its fitness. Fitness can be defined quantitatively by an organism's basic reproductive rate $\left(R_{0}\right)$. The size of $R_{0}$ is given by the ratio of the number of surviving progeny to the number of founder cells of the bacterial population. Clearly, when $R_{0}$ falls below unity, any given bacterial population will decline and will ultimately be extinguished. The higher the value of $R_{0}$, the fitter are the bacteria that constitute the population. The strategies evolved by bacterial pathogens to attain $R_{0}>1$ are often captured in a metaphor which refers to their genetic intelligence, a reflection of how evolution, over time, effects a balance between the mechanisms of genome variation and genome stability. Natural selection acts so as to trade off genome stability against genome variation to achieve fitness, a reflection of the immense power of natural selection, apparently purposeful, but in fact blind. This evolution occurs over very long periods of time, during which the content and organization of bacterial genomes are shaped by loss and gain of DNA. There are no species barriers to genetic exchange in bacteria as there are, for example, in mammals: bacterial sex through recombination is uncoupled from replication. Over shorter time periods (hours, weeks or months) within-host adaptive behaviour of bacterial populations is largely determined by intragenomic gene variation and changes in gene activity rather than acquisition of novel DNA.

\section{Population biology of $\boldsymbol{H}$. influenzae}

From an ancestral bacterium, rare mutations occur in progeny cells but if acquisition of DNA by lateral transfer events does not occur, then over time the species population retains a clonal structure. $\mathrm{Hi}$ is one of several commensal pathogens that are naturally transformable and this, as well as mechanisms such as conjugation and transduction, can result in lateral transfer of DNA. Acquisition of small DNA sequences, much less than the size of a gene, but also larger elements such as ICE Hin 1056 and pathogenicity islands (Mohd-Zain et al., 2004), have been well documented. Such DNA acquisitions may enhance fitness and this selective advantage increases the frequency distribution of particular genotypes in the population. The population structure is affected not only by these intergenomic DNA transfers but also by periodic extinctions of genotypes that are not fit (most DNA changes are deleterious) or through population bottlenecks associated with human-to-human transmission. Thus, constructing the long-term phylogeny of $\mathrm{Hi}$ over time is complicated by recombination and extinctions and is 
reflected in the non-clonal (panmictic) population structure of the $\mathrm{Hi}$ species. Although strains elaborating each of the six capsular polysacchides exhibit a more clonal population structure, perhaps reflecting their shorter evolutionary time frame (Musser et al., 1988), multilocus enzyme electrophoresis, ribotyping and multilocus sequence typing each independently indicate that the majority of $\mathrm{Hi}$ strains have undergone frequent recombination (Meats et al., 2003). In one analysis of a collection of carriage and disease isolates there was abundant evidence of recombination based on mosaic sequences, linkage equilibrium between loci and a lack of congruence between gene trees (Cody et al., 2003). More recently, further insights into the composition, organization and variation of $\mathrm{Hi}$ have been obtained through complete genome sequences of many different strains. The DNA of these different genotypes comprises core sequences (found in all strains of the species), dispensable sequences (those found in more than one strain but absent from one or more strains) and unique sequences (found in only one strain). For each new genome sequenced, some $2 \%$ of sequences fall into the unique category, indicating that the species $\mathrm{Hi}$ has an 'open' genome of unlimited and cumulative novelty as distinct from species in which the genome sequences of a subset of strains define the pangenome (Medini et al., 2005).

\section{$H$. influenzae adapts to its host through gene regulation and gene variation}

Adaptation of microbes to their host environment involves two genetic mechanisms: gene regulation or gene variation (Hood \& Moxon, 2002). A substantial proportion of research on bacterial pathogens in the past two to three decades has been devoted to investigating how microbes detect molecular signals emanating from host tissues and how these are transduced so as to effect adaptive phenotypic changes to the population of bacterial cells. Factors such as osmolarity, $\mathrm{pH}$ and available nutrients influence pathogenic potential by affecting the production of virulence determinants and by controlling growth rate. For example, upon contact with a host cell, many bacterial pathogens increase the transcription of virulence genes in a deterministic fashion. Indeed, it has been proposed that expression of virulence factors may depend upon the microbial population reaching a certain density so as to trigger the elaboration of signalling molecules e.g. $\mathrm{N}$ acylhomoserine lactones (Williams, 2007).

Complete genome sequences of several $\mathrm{Hi}$ genomes indicate that $\mathrm{Hi}$ has limited potential for adaptation through classic signalling systems such as two-component histidine kinases and does not possess homologues of genes for quorum sensing (Fleischmann et al., 1995). However, there is a striking number of hypermutable DNA sequences, predominantly tetranucleotide repeats (Hood et al., 1996b), which undergo slipped-strand mispairing so as to generate phenotypic diversity through frame-shifts in genes, for example those involved in lipopolysaccharide (LPS) biosynthesis and iron acquisition. In addition, there are at least two examples of tandem DNA repeats that modulate transcription of the fimbrial (van Ham et al., 1993) and HMW adhesins (Dawid et al., 1999). These genetic loci, many of which exhibit phase variation (highfrequency, reversible on-to-off/off-to-on switching) have been called contingency loci to emphasize their potential to enable at least a few bacteria in a given population to adapt to the unpredictable and precipitous changes in the host environment (Moxon et al., 1994). These combinatorial variations in phenotype have major implications for both commensal and virulence behaviour through their effects on microbial-host adherence, immune evasion and acquisition of nutrients. There is also evidence for gene regulation through variable methylation mediated by DNA repeats located in a type III restriction-modification system (Srikhanta et al., 2005).

\section{$H$. influenzae LPS is a determinant of commensal and virulence behaviour}

The role of LPS in the adaptive behaviour of $H i$ is an exemplar of the interplay of gene regulation and gene variation in microbial-host interactions and has important consequences for commensal and virulence behaviour. LPS is the most prevalent component of the Hi cell envelope and confers important properties on the outer membrane. It facilitates the influx of essential nutrients while acting as a barrier to many potentially toxic molecules. It is essential for the efficient surface assembly of proteins that control, for example, cation or anion channels. LPS is also implicated in every phase of $\mathrm{Hi}$ pathogenesis: colonization, evasion of host clearance mechanisms (innate and acquired) and tissue injury. The availability of the whole genome sequence of $\mathrm{Hi}$ strain $\mathrm{Rd}$ in 1995 (Fleischmann et al., 1995) facilitated enormously progress on the genetics of LPS biosynthesis of this commensal pathogen. The majority of episodes of the important and common disease otitis media are caused by capsule-deficient (non-typable: NT) $H i$ strains. The genome sequence of strain Rd allowed identification of core biosynthetic genes, for example those required for making lipid $\mathrm{A}$ and conserved core sugars (inner core) as well as the glycosyltransferases responsible for the highly variable outer core sugars, such as sialic acid, and several non-sugar additions such as phosphoethanolamine, phosphorylcholine, glycine and acetate. The sequenced genome of strain Rd indicated that the content and organization of the LPS genes of $\mathrm{Hi}$ are sporadically distributed compared to the more discrete clustering of LPS biosynthetic genes typical of the Enterobacteriaceae (Hood et al., 1996a). The legacy of LPS biosynthetic gene discovery through genomics, together with the construction of defined mutants, opened the door to obtaining fine-structural details of LPS and revealed the extraordinary heterogeneity of LPS glycoforms that are a hallmark of $\mathrm{Hi}$ (Schweda et al., 2007). This variability is mostly attributable to diversity of outer core sugars and 
substituents of the inner core. The inner core consists of lipid A, a single phosphorylated Kdo (2-keto-3-deoxyoctonic acid; systematic name 3-deoxy-D-manno-2-octulosonic acid) and a conserved triheptose backbone (Fig. 1). A detailed analysis of an epidemiologically diverse collection of encapsulated and NT strains of $\mathrm{Hi}$, made up of both carriage and disease (otitis media) isolates (Cody et al., 2003), has shown that this LPS diversity is the result of several mechanisms: allelic variation, the acquisition of distinct transferases, phase variation and non-stoichiometric additions of molecules such as phosphorylcholine. A particular challenge lies in determining the biological relevance of this LPS variation.

\section{Sialylated LPS glycoforms are a virulence determinant of $\boldsymbol{H}$. influenzae}

To illustrate the importance of understanding how knowledge of LPS structure informs biological function, I now turn to the role of sialylated glycoforms as an exemplar. When mutations in genes required for sialylation of LPS were introduced into several genetically distinct NT $\mathrm{Hi}$ and these mutants were compared to the wild-type in an animal model (chinchillas) of acute otitis media, drastic attenuation was observed (Bouchet et al., 2003). Further, this attenuation occurred in strains where the sialylated glycoforms of the wild-type were different. For example, in strain 486, Neu5Ac is found as a monosialylated glycoform, whereas in contrast, in strain 375 , Neu5Ac is present in the di-sialylated form (Fig. 1). The mechanism of LPS sialylation in pathogenicity was investigated and it was shown that Neu5Ac interferes with the binding, activation and immune clearance of $\mathrm{Hi}$ effected by complement components (Figueira et al., 2007). However, a further key finding was that sialylation of $\mathrm{Hi}$ LPS is apparently dependent upon scavenging the required precursors from the host because the genes for the early steps in sialic acid biosynthesis in $H i$ are absent (Vimr et al., 2000). As well as being a component of the LPS of all strains for which detailed structure is known, Neu5Ac is a potential carbon

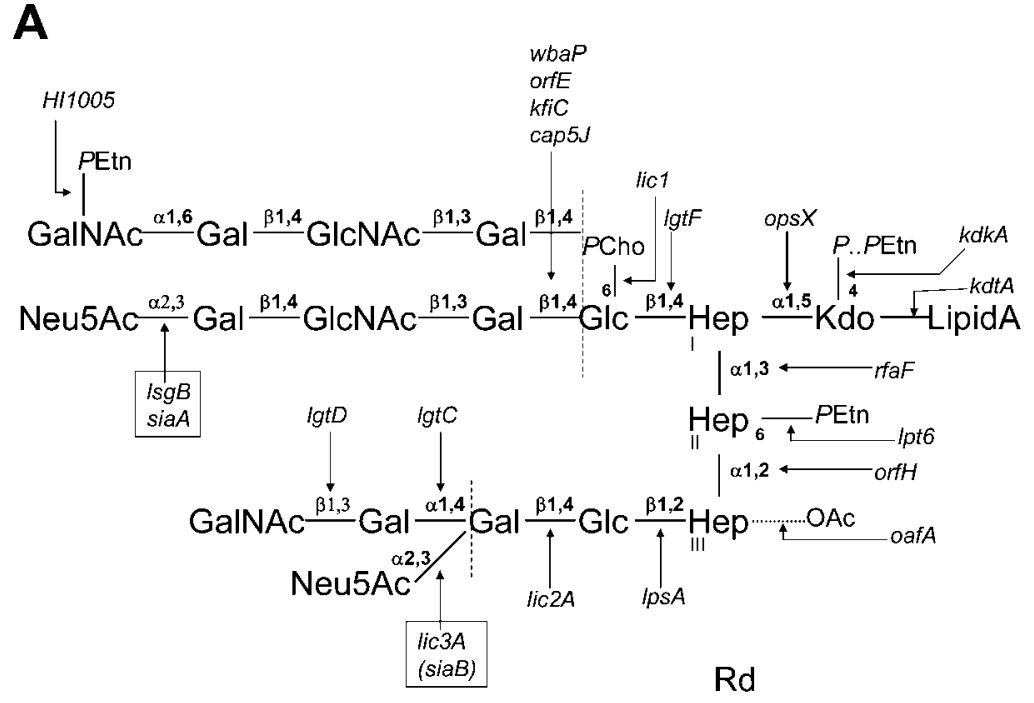

B
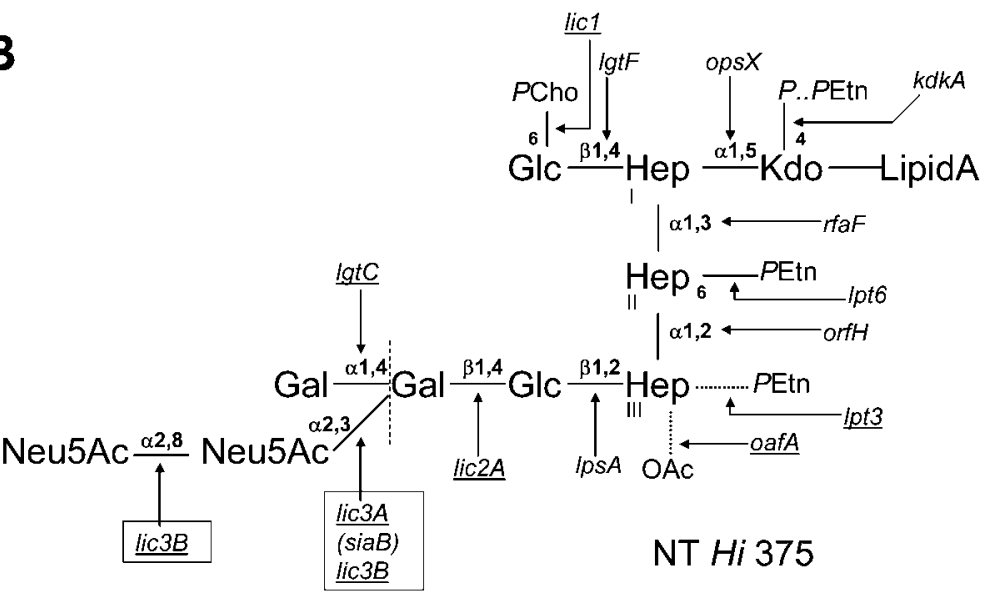

Fig. 1. Schematic representation of the fully extended LPS structure of $\mathrm{Hi}$ strains $\mathrm{Rd}$ and NT Hi 375 following growth in the presence of sialic acid. Panel A shows the genetic blueprint for biosynthesis for strain Rd and panel B shows how this information was used to develop and then confirm the corresponding data for strain 375 . The genes encoding the biosynthetic enzymes responsible for adding the components are indicated by an arrow at the appropriate linkage. Sialometabolism genes are boxed. The vertical dashed lines indicate positions in the LPS at which alternative substitutions, as indicated to the left of the line, can be included. $w b a P$, orfE, $k f i C$ and cap5J each result in loss of the complete tetrasaccharide unit when mutated. Sialic acid can be included as sialyllactose extended from Heplll and as the sialylated lacto- $N$-neotetraose (sINnt) epitope extended from Hepl. In panel $\mathrm{B}$, phase variable genes contributing to LPS heterogeneity are underlined. 
and energy source (Vimr et al., 2000). It has been shown that $\mathrm{Hi}$ acquires Neu5Ac during experimental infection of chinchillas, making it a potential source of carbon and energy to the bacterium in vivo (Bouchet et al., 2003). So, how does Neu5Ac contribute to the fitness of $\mathrm{Hi}$ ? After all, I have argued that although it is important to understand the molecular mechanisms underlying pathogencity, this proximate insight must also be consistent with an ultimate contribution to fitness. This is the major aim of our present investigations. In particular, at least one of the several sialyltransferases (Lic3) is phase variable (Hood et al., 2001), suggesting that there is a selective advantage to $\mathrm{Hi}$ if it can hedge its bets on whether it sialylates its LPS $\left(\mathrm{Sia}^{+}\right.$or phase $\mathrm{ON}$ ) or not $\left(\mathrm{Sia}^{-}\right.$or phase $\left.\mathrm{OFF}\right)$. A reasonable hypothesis is as follows. Colonization of the nasopharynx would be favoured by $\mathrm{Sia}^{-}$organisms, since the more negatively charged surface of $\mathrm{Sia}^{+}$organisms would downregulate interactions with host epithelial cells. However, virus infections leading to inflammation (nasopharyngitis) are a common event and under these circumstances, the $\mathrm{Sia}^{+}$organisms would have a fitness advantage since their clearance would be reduced as in the chinchilla studies cited above. Experiments are needed to confirm or reject this hypothesis, in which the evolution of variable sialylation and the propensity of sialylated glycoforms to cause diseases are traded off against the fitness advantage to the organism's survival.

The generation of population diversity through mutation is an important source of variants upon which selection can act to increase fitness, but as a strategy it is inherently wasteful; most variants are not selected. In contrast, gene regulation offers a prescriptive strategy for adaptation. When changes are encountered in the environment, bacterial cells can sense these changes and bring about a coordinated change in gene activity. The regulation of genes for sialometabolism in $\mathrm{Hi}$ affords an exemplar of this form of host adaptation. We used a whole-genome microarray to investigate the interplay between utilization of Neu5Ac for catabolism and sialylation of LPS (Randle et al., 2005). When we compared the transcriptome of $\mathrm{Hi}$ that was grown in either the absence or the presence of an exogenous source of Neu5Ac, we found that the presence of Neu5Ac downregulated the transcription of a set of nine contiguous genes (designated the sialometabolism locus). The sialometabolism locus is organized such that six genes are transcribed in one direction (five genes required for Neu5Ac catabolism and one regulatory gene) and three genes are transcribed in the opposite direction. Two of these code for proteins of a tripartite ATP-independent perisplasmic (TRAP) transporter (Severi et al., 2005) and one gene (HI048) is a homologue of Escherichia coli NanM, a Neu5Ac mutarotase (Severi et al., 2008). Between the divergently transcribed catabolism and transport genes, there is an intergenic, non-coding region (353 bp) containing a consensus CRP (catabolite repressor protein) binding site (Randle et al., 2005; Johnston et al., 2007). The transcriptional downregulation of both transporter and catabolic genes, observed using DNA microarrays, was confirmed and quantified by q-PCR and indicated an important signalling role for Neu5Ac in the regulation of sialometabolism. Indeed, the sialometabolism locus is highly conserved in $\mathrm{Hi}$ strains despite the well-documented inter-strain variability in other genes that determine the location of Neu5Ac on the glycoform extensions from the three heptoses that make up the backbone of the Hi LPS core (Schweda et al., 2007).

The mechanism(s) by which exogenous Neu5Ac alters transcriptional activity is not yet undertood. Given that $\mathrm{Hi}$ lacks nanR, the gene that encodes a negative transcriptional regulator of sialometabolism genes from the nanA promoter in E. coli, siaR has been thoroughly investigated (Johnston et al., 2007). SiaR possesses a helix-turn-helix DNA-binding domain, a characteristic associated with regulatory proteins, and binds to the intergenic region. Based on RT-PCR and qPCR, disruption of SiaR increases transcription of both uptake and catabolic genes of the sialometabolism locus. It was found that mutation of siaR resulted in up to a 19-fold increase in expression of sialometabolism genes (Derek Hood, personal communication). A reasonable hypothesis is that the SIS domain present in the SiaR protein could be a binding site for Neu5Ac or perhaps other related sugars (e.g. $\mathrm{N}$-acetylglucosamine or glucosamine 6-phosphate) that activate(s) the repressor activity of SiaR. In contrast to SiaR, which affects both catabolic and transporter genes, CRP is a positive transcriptional regulator of Neu5Ac uptake, but has much less effect upon the catabolic genes. The more relevant selection pressures contributing to the evolution of LPS sialylation and its regulation are likely to be a function of $\mathrm{Hi}$ fitness for carriage and transmission rather than its role in disease.

\section{Prevention of $\boldsymbol{H}$. influenzae diseases by immunization}

From the public health perspective, $\mathrm{Hi}$ diseases are a major cause of mortality and morbidity, especially in young children (Moxon \& Murphy, 2000). As emphasized earlier, type b strains cause serious invasive (bacteraemic) infections, including meningitis. One of the success stories of modern vaccinology has been the development of successful polysaccharide-protein conjugate vaccines that induce protective antibodies to the type $\mathrm{b}$ capsule. These vaccines not only prevent $\mathrm{Hi}$ type b disease, even in very young infants, but also confer indirect protection by reducing carriage (herd immunity). Important factors in the success of these conjugate vaccines are that serotype b strains (those that have a capsular polysaccharide composed of ribosyl-ribitol phosphate) exercise a virtual monopoly in the epidemiology of life-threatening $\mathrm{Hi}$ bacteraemic infections, that this capsular polysaccharide is necessary for the heightened virulence potential of $\mathrm{Hi}$ and that the key protective epitopes of the capsule are conserved.

However, capsule-deficient, or non-typable (NT) Hi are also a major cause of upper (e.g. otitis media) and lower 
(pneumonia) respiratory tract infections in all parts of the world (Murphy, 2003). In particular, acute lower respiratory infections are a major killer in the very young in socio-economically deprived countries and otitis media is the commonest reason for the prescription of antibiotics in developed nations. What about a vaccine against NT Hi diseases? Sialic acid might seem to be an obvious candidate, arguing along parallel lines to the success of vaccines based on the type $\mathrm{b}$ capsular antigen. But there are compelling reasons why Neu5Ac will not be a potentially successful vaccine candidate. In contrast to the type $b$ antigen, the conformation and location of Neu5Ac is highly variable (Fox et al., 2006; Hood et al., 1999; Jones et al., 2002; Schweda et al., 2007). A further serious drawback is the potential mimicry of Neu5Ac of host glycoproteins and glycolipids (Schweda et al., 2007). Vaccine development requires a judicious appraisal of virulence and variation. Thus, I will end where I began: Fred Griffith '...believed that a proper understanding of epidemiological problems could come only from more detailed and discriminating knowledge of infectious bacterial species, and of the nature of bacterial virulence and variation'.

\section{Acknowledgements}

The author wishes to recognize the many contributions of the Molecular Infectious Diseases Group in the Weatherall Institute of Molecular Medicine, University of Oxford, especially Dr Derek Hood. The research depended on collaborations with Jim Richards and his Research Group (National Research Council of Canada), Elke Schweda (Karolinska Institute, Huddinge, Sweden) and Steven Pelton's Research Group at the Maxwell Finland Laboratories, Boston University, USA.

\section{References}

Alexander, H. E. \& Leidy, G. (1953). Induction of streptomycin resistance in sensitive Haemophilus influenzae by extracts containing desoxyribonucleic acid from resistant Haemophilus influenzae. J Exp Med 97, 17-31.

Bouchet, V., Hood, D. W., Li, J., Brisson, J. R., Randle, G A., Martin, A., Li, Z., Goldstein, R., Schweda, E. K. \& other authors (2003). Hostderived sialic acid is incorporated into Haemophilus influenzae lipopolysaccharide and is a major virulence factor in experimental otitis media. Proc Natl Acad Sci U S A 100, 8898-8903.

Carithers, H. A. (1974). The first use of an antibiotic in America. Am J Dis Child 128, 207-211.

Cody, A. J., Field, D., Feil, E. J., Stringer, S., Deadman, M. E., Tsolaki, A. G., Gratz, B., Bouchet, V., Goldstein, R. \& other authors (2003). High rates of recombination in otitis media isolates of non-typeable Haemophilus influenzae. Infect Genet Evol 3, 57-66.

Dawid, S., Barenkamp, S. J. \& St Geme, J. W., 3rd (1999). Variation in expression of the Haemophilus influenzae HMW adhesins: a prokaryotic system reminiscent of eukaryotes. Proc Natl Acad Sci U S A 96, 1077-1082.

Figueira, M. A., Ram, S., Goldstein, R., Hood, D. W., Moxon, E. R. \& Pelton, S. I. (2007). Role of complement in defense of the middle ear revealed by restoring the virulence of nontypeable Haemophilus influenzae siaB mutants. Infect Immun 75, 325-333.
Fleischmann, R. D., Adams, M. D., White, O., Clayton, R. A., Kirkness, E. F., Kerlavage, A. R., Bult, C. J., Tomb, J. F., Dougherty, B. A. \& other authors (1995). Whole-genome random sequencing and assembly of Haemophilus influenzae Rd. Science 269, 496-512.

Fox, K. L., Cox, A. D., Gilbert, M., Wakarchuk, W. W., Li, J., Makepeace, K., Richards, J. C., Moxon, E. R. \& Hood, D. W. (2006). Identification of a bifunctional lipopolysaccharide sialyltransferase in Haemophilus influenzae: incorporation of disialic acid. J Biol Chem 281, 4002440032.

Hayes, W. (1966). Genetic transformation: a retrospective appreciation. J Gen Microbiol 45, 385-397.

Hood, D. \& Moxon, E. R. (2002). Gene variation and gene regulation in bacterial pathogenesis. In Signals, Switches, Regulons and Cascades: Control of Bacterial Gene Expression, pp. 19-40. Edited by D. A. Hodgson \& C. M. Thomas. Cambridge: Cambridge University Press.

Hood, D. W., Deadman, M. E., Allen, T., Masoud, H., Martin, A., Brisson, J. R., Fleischmann, R., Venter, J. C., Richards, J. C. \& Moxon, E. R. (1996a). Use of the complete genome sequence information of Haemophilus influenzae strain Rd to investigate lipopolysaccharide biosynthesis. Mol Microbiol 22, 951-965.

Hood, D. W., Deadman, M. E., Jennings, M. P., Bisercic, M., Fleischmann, R. D., Venter, J. C. \& Moxon, E. R. (1996b). DNA repeats identify novel virulence genes in Haemophilus influenzae. Proc Natl Acad Sci U S A 93, 11121-11125.

Hood, D. W., Makepeace, K., Deadman, M. E., Rest, R. F., Thibault, P., Martin, A., Richards, J. C. \& Moxon, E. R. (1999). Sialic acid in the lipopolysaccharide of Haemophilus influenzae: strain distribution, influence on serum resistance and structural characterization. Mol Microbiol 33, 679-692.

Hood, D. W., Cox, A. D., Gilbert, M., Makepeace, K., Walsh, S., Deadman, M. E., Cody, A., Martin, A., Månsson, M. \& other authors (2001). Identification of a lipopolysaccharide alpha-2,3-sialyltransferase from Haemophilus influenzae. Mol Microbiol 39, 341-350.

Johnston, J. W., Zaleski, A., Allen, S., Mootz, J. M., Armbruster, D., Gibson, B. W., Apicella, M. A. \& Munson, R. S., Jr (2007). Regulation of sialic acid transport and catabolism in Haemophilus influenzae. Mol Microbiol 66, 26-39.

Jones, P. A., Samuels, N. M., Phillips, N. J., Munson, R. S., Jr, Bozue, J. A., Arseneau, J. A., Nichols, W. A., Zaleski, A., Gibson, B. W. \& Apicella, M. A. (2002). Haemophilus influenzae type b strain A2 has multiple sialyltransferases involved in lipooligosaccharide sialylation. J Biol Chem 277, 14598-14611.

McCarty, M. (1985). The Transforming Principle: Discovering that Genes are Made of DNA. New York: W. W. Norton \& Co.

Meats, E., Feil, E. J., Stringer, S., Cody, A. J., Goldstein, R., Kroll, J. S., Popovic, T. \& Spratt, B. G. (2003). Characterization of encapsulated and noncapsulated Haemophilus influenzae and determination of phylogenetic relationships by multilocus sequence typing. J Clin Microbiol 41, 1623-1636.

Medini, D., Donati, C., Tettelin, H., Masignani, V. \& Rappuoli, R. (2005). The microbial pan-genome. Curr Opin Genet Dev 15, 589594.

Mohd-Zain, Z., Turner, S. L., Cerdeno-Tarraga, A. M., Lilley, A. K., Inzana, T. J., Duncan, A. J., Harding, R. M., Hood, D. W., Peto, T. E. \& Crook, D. W. (2004). Transferable antibiotic resistance elements in Haemophilus influenzae share a common evolutionary origin with a diverse family of syntenic genomic islands. J Bacteriol 186, 8114-8122.

Moxon, E. R. \& Murphy, T. F. (2000). Haemophilus influenzae. In Mandell, Douglas and Bennett's Principles and Practice of Infectious Diseases, 5th edn, pp. 2369-2378. Edited by G. L. Mandell, J. E. Bennett \& R. Dolin. Philadelphia, PA: Churchill Livingstone. 
Moxon, E. R. \& Vaughn, K. (1981). The tybe b capsular polysaccharide as a virulence determinant of Haemophilus influenzae: studies using clinical isolates and laboratory transformants. J Infect Dis 143, 517524.

Moxon, E. R., Rainey, P. B., Nowak, M. A. \& Lenski, R. E. (1994). Adaptive evolution of highly mutable loci in pathogenic bacteria. Curr Biol 4, 24-33.

Murphy, T. F. (2003). Respiratory infections caused by non-typeable Haemophilus influenzae. Curr Opin Infect Dis 16, 129-134.

Musser, J. M., Kroll, J. S., Moxon, E. R. \& Selander, R. K. (1988). Clonal population structure of encapsulated Haemophilus influenzae. Infect Immun 56, 1837-1845.

Randle, G. A., Hood, D. W., Makepeace, K. \& Moxon, E. R. (2005). The role of sialic acid in the pathogenesis of Haemophilus influenzae. Abstract A63, ASM Pasteurellaceae 2005 Meeting, Hawaii, USA.

Schweda, E. K., Richards, J. C., Hood, D. W. \& Moxon, E. R. (2007). Expression and structural diversity of the lipopolysaccharide of Haemophilus influenzae: implication in virulence. Int J Med Microbiol 297, 297-306.

Severi, E., Randle, G., Kivlin, P., Whitfield, K., Young, R., Moxon, R., Kelly, D., Hood, D. \& Thomas, G. H. (2005). Sialic acid transport in Haemophilus influenzae is essential for lipopolysaccharide sialylation and serum resistance and is dependent on a novel tripartite ATP-independent periplasmic transporter. Mol Microbiol 58, 1173 1185.
Severi, E., Muller, A., Potts, J. R., Leech, A., Williamson, D., Wilson, K. S. \& Thomas, G. H. (2008). Sialic acid mutarotation is catalyzed by the Escherichia coli $\beta$-propeller protein YjhT. J Biol Chem 283, 48414849.

Smith, W., Andrews, C. \& Laidlaw, P. P. (1933). A virus obtained from influenza patients. Lancet ii, 66-68.

Srikhanta, Y. N., Maguire, T. L., Stacey, K. J., Grimmond, S. M. \& Jennings, M. P. (2005). The phasevarion: a genetic system controlling coordinated, random switching of expression of multiple genes. Proc Natl Acad Sci U S A 102, 5547-5551.

van Ham, S. M., van Alphen, L., Mooi, F. R. \& van Putten, J. P. (1993). Phase variation of $H$. influenzae fimbriae: transcriptional control of two divergent genes through a variable combined promoter region. Cell 73, 1187-1196.

Vimr, E., Lichtensteiger, C. \& Steenbergen, S. (2000). Sialic acid metabolism's dual function in Haemophilus influenzae. Mol Microbiol 36, 1113-1123.

Williams, P. (2007). Quorum sensing, communication and crosskingdom signalling in the bacterial world. Microbiology 153, 39233938.

Zwahlen, A., Kroll, J. S., Rubin, L. G. \& Moxon, E. R. (1989). The molecular basis of pathogenicity in Haemophilus influenzae: comparative virulence of genetically-related capsular transformants and correlation with changes at the capsulation locus cap. Microb Pathog 7, 225-235. 